\title{
When Big Data Becomes Little Data: Predicting Freshman Attrition
}

\author{
Joseph B. O’Donnell and Paul L. Sauer
}

Joseph B. O’Donnell

Department of Accounting

Canisius College

2001 Main Street,

Buffalo, New York 14208

Email:odonnelj@canisius.edu

Phone: (716) 888-2867

Paul L. Sauer

Marketing and Information Systems Department

Canisius College

2001 Main Street,

Buffalo, New York 14208

Email:sauer@canisius.edu Phone: (716) 888-2631

\begin{abstract}
The study involves a college that evolved from an extensive attrition prediction model with a robust dataset to a trimmed down
\end{abstract}




\section{The BRC Academy Journal of Education Vol. 8, No. 1}

model with a sparse dataset. This paper the compares effectiveness of this parsimonious model with an extensive model in predicting attrition. Authors find that the trimmed down model possesses improved predictive capability for persisters but lower predictive accuracy for dropouts and students overall than the extensive model. Study results surprisingly suggest that student loan amounts and being commuter students are positively associated with persistence, while SAT verbal/reasoning score is negatively associated with persistence. Authors investigate use of pooled annual data versus split training and confirmation data samples in testing the attrition model. The study offers insight to educational researchers and administrators on the predictive sufficiency of using a relatively small set of easy to attain student background and academic variables in predicting attrition.

Keywords: retention, methodology, persistence, financial need, logit, student characteristics

DOI: https://dx.doi.org/10.15239/j.brcacadje.2020.08.01.ja01

\section{INTRODUCTION}

Student retention rates significantly affect the image and financial health of many colleges and universities. "Higher the retention rate, more likely for the institution to be ranked higher, secure more federal funds, traded favorably for appropriation and have easier path to program accreditations" (Thammasiri, Delen, Meesad, \& Nihat 2014, p. 321). Potentially, high student retention is most important to small private colleges that more heavily depend upon tuition for subsistence. As some small private colleges face declining enrollments, administrators ramp up efforts to retain current students in hopes of preserving the financial health of those colleges. This study describes how the loss of a welldeveloped model of attrition eventually led to a lean predictive model of attrition and evaluates the effectiveness of the resulting predictive model. One challenge for many institutions is limited capacity to gather accurate student engagement and behavioral data and connect them to 
the student information system for inclusion in predictive models" (Burke, Parnell, Wesaw \& Kruger, 2017, p.3). The study offers valuable insights for administrators on the importance of maintaining attrition data despite limited resources and offers a potential economical approach for resource strapped schools. This study adds to the student retention literature by investigating the balance of maintaining an effective attrition model with the constraints of limited resources to support the model.

The research reported in this paper was conducted at a small, private liberal arts and sciences college (henceforth, called the "Target College") located in the northeastern U.S. At one time Target College had a comprehensive attrition model with an extensive database that over time deteriorated into a lean model with a sparse database. Target College experienced a steady $22 \%$ decline in freshman enrollment from 2012 to 2017. In light of this declining enrollment, the need for data to forecast attrition grew significantly. Unfortunately, the only remaining available data for predicting the likelihood of each freshman student dropping out of college by the beginning of their sophomore year consisted of a limited number of primarily administrative variables. This limited number of variables resulted from administrative personnel decisions and other organizational changes, such as attrition of research team members. The once robust database that included student characteristic variables and a rich set of matriculating freshman self- report items became a database with only background student characteristic variables, such as high school GPA, SAT scores, and demographic information including gender and race. Offering some hope, a previous Astin (1997) study found that a trimmed down database of only four student background variables explained the greatest amount of variance in attrition. Describing the development and decline of Target College's attrition model provides insight on the precarious status of models that compete for resources in the competitive small private college environment. 


\section{The Bigger Data Period - Variable Growth and Model DeVELOPMENT}

In the 1970s, Target College began predicting attrition and intervening with freshman at risk of attrition. To gain a better understanding of reasons for student attrition, Target College researchers worked to develop an improved measurement instrument based on an expanded data set. The resulting measurement instrument included an amalgam of items extracted from a variety of established external instruments supplemented by items from a series of ad-hoc in-house studies. External instrument items originated from the study of freshmen portion of the Cooperative Institutional Research Program (CIRP) and the College Student Experiences Survey from the Higher Education Research Institute, UCLA Graduate School of Education. Ad-hoc measures included an assortment of items used in several studies dealing with specific issues, such as the effect of alcohol use on student attrition. Measures originated from several different situations including college senior year surveys and student focus groups. Freshmen completed the resulting survey instrument as part of the matriculation process. When combined with the internally generated background data, the resulting data set provided insights into possible reasons for attrition that a student background-based data set alone could not detect.

During the period from 1995 to 2005, Target College's measurement instrument grew to include the matriculating freshman self-response scales combined with administratively obtained student data from the application and enrollment forms. As a result, this college maintained a rich array of both unobtrusive background generated measures such as SAT scores and parent's income combined with the self-report measures. These measures provided insights into underlying reasons and motives for student behavior and performance. After establishing this rich dataset, researchers turned their efforts towards identifying an optimal approach to testing the model of attrition. 
During this period, the statistical techniques employed evolved from simple regression models to more sophisticated two-stage models, logit models and structural equation models (SEMs), that provided a richer understanding of attrition and probabilities of attrition for each student. In a similar way, Stage (1998) used two stage models to identify at-risk freshmen for college staff intervention. Freshmen self-report measures significantly improved predictability of attrition in these models.

\section{How Big Data Became Little Data - Institution and Data Constraints}

In 2001, one of the co-authors of this paper joined Target College's research team to assist in further developing and refining the attrition model. The team consisted of a team leading faculty member, a senior college administrator and the co-author. At that time, the variety of variables available in the dataset consisted of the richest set ever compiled at Target College. This team leader retired from the faculty in 2005. Just before retiring, this faculty member reduced the number of measures on the matriculating freshmen self-report items from 79 to 37 . The 37 retained items displayed a significant correlational relationship to one of the 12 latent factors (constructs) generated by exploratory factor analysis (EFA) used to generate factor scores for the logit attrition model.

The senior administrator became less involved in research team projects. Soon after 2005, he left Target College to take an administrative position at another university. The faculty member, who joined the research team in 2001, now assumed sole responsibility at Target College for forecasting attrition. The resulting attrition model provided limited guidance to college counselors for student interventions. The logit model identified high dropout risk students and a college administrator, who was not part of the research team, performed intervention interviews with these high-risk students. 
Two years later, this administrator left the college and the Target College hired an institutional researcher who took over management of the model and the task of predicting a student's likelihood of attrition. The institutional researcher and the faculty member now constituted the attrition research team. Eventually, the institutional researcher assumed sole responsibility for maintaining and running the model. During this period, the Target College streamlined freshmen orientation activities and ended the use of the freshmen self-report survey. A sparse database and a lean attrition model resulted from these changes. In summary, significant Target College organizational and environmental changes following the departure of the research team members resulted in big data becoming little data (See Table 1).

Beginning around 2012, freshman enrollments began to consistently decline at a substantial rate. As a result, Target College raised its focus on reducing student attrition and hired a third party firm to provide attrition forecasts. Within a year or two after Target College convinced the remaining faculty member, from the former attrition research team, to assist in forecasting attrition. Review of the third party model suggested that it employed a combination of logit model and decision-tree model similar to the chi-square automatic interaction detector (CHAID) to forecast attrition. The logit model, however, was the primary model used by the third party in predicting individual student probability of attrition. The logit model successfully predicted attrition in the big data years prior to 2006 and was easy to set up and run. Since 2013, Target College resumed using the logistic regression attrition model but with the sparse dataset. On a year-by-year basis, this attrition model provides reasonably good results. See Table 2 for a timeline of the organizational periods for forecasting retention. 


\section{Going Forward with Sparse (SMall) Data}

Given the goal of improving the predictive performance of Target College's logit model when applied to its most recent 2013-2017 sparse dataset, we seek to determine:

1.) How well our data-sparse model performs when applied to data from the 2013 to 2017 datasets that include background student data only,

2.) which statistical modeling approach is a good fit for the attrition prediction model, and

3.) whether pooling the 2013 to 2017 data or splitting the data into training and confirming datasets is merited for our study.

\section{Literature Review: Searching for a Model to Predict Attrition}

In reviewing the literature, we found that the pattern of model development regarding freshman attrition evolved from early efforts to establish a descriptive analysis providing a theoretical foundation to later efforts to identify variables and constructs, which offered valid measures that improved predictability (Herzog, 2005). Theoretically based models of attrition began to appear in the 1970s (Astin, 1972; Bean, 1982; Tinto, 1975). For an excellent review of development of these models, see Aljohani (2016). Studies that followed included efforts to either compare theories (Milem \& Berger, 1997) or integrate theories (Cabrera, Nora \& Castaneda, 1993) to explain attrition occurring after the freshman year. These studies also addressed the predictive factors that included funding issues, such as grants and other forms of financial aid (Reason 2003). Herzog (2005) considered whether the student leaves to transfer to another school or drops out of college completely. Attrition model categories used in this review include descriptive theoretical, normative and predictive models. Descriptive models arise from psychological theories, normative models 
revolve around sociological theories, and predictive models focus on students' characteristics and background in predicting attrition.

\section{Descriptive Theoretical Models}

The more sophisticated and complex models of student attrition include an extensive array of variables to describe the causes of student attrition (Glynn, Sauer \& Miller, 2003). As noted by Aljohani (2016) the early attrition models focused on development of primarily psychological theory but not sociological theory. The early attrition models appealed to those academicians and college administrators who sought to understand and describe the root psychological causes of student attrition. The goal of such efforts was to determine students' underlying internal psychological processes and other influential factors that lead to a better understanding of student attrition behavior. The attrition models that fall in the descriptive theoretical category include Astin's (1984) model based on the psychological perspective of involvement, Bean's (1982) model based on the theory of reasoned action (Fishbein \& Ajzen, 1975), and Tinto's (1975) and Spady's (1970) models based on Durkheim's (2005) model of suicide. The results and findings led to the revelation that students who avoid dropping out their freshman year and persist to graduation are initially more socially integrated into elements of the student community than students who have dropped out (Bean \& Metzner, 1985; Billson \& Brooks-Terry, 1987; Hausmann, Schofield \& Woods 2007; Pascarella \& Terenzini 1983; Tinto, 1975).

Subsequent research proposed that adding variables to these descriptive models might enable researchers to transform these models into normative models that guide intervention efforts needed to reduce attrition. The added variables may play a significant antecedent, mediational or moderating role in altering the size of the impact on attrition (Kovacic, 2010; Reason, 2003). The next section discusses normative models. 


\section{Normative Models}

The foundations of normative models are researcher findings that social norms are essential to understanding human behavior (Cialdini, Reno \& Kallgren, 1990; McKirnan, 1980; Oyserman, \& Lee, 2008). Roland, Frenay, and Boudrenghien, (2018) examined the relationship of social norms with student attrition, in the context of the theory of planned behavior (Ajzen, 1991). Normative attrition models also focus on professional counselors' interventions that potentially offer opportunities for significantly reducing the attrition rate. To be successful counselors need to understand the underlying motives, attitudes, perceptions, drives, stimuli, satisfaction and intent that represent the psychological and sociological forces leading to attrition (Sparkman, Maulding \& Roberts, 2012). By adding and testing the effect of these factors in a series of simulations using a behavioral model, the counsellor will gain insight and be able to direct attention to the factors with the greatest likelihood of reducing attrition (Soria, Laumer, Morrow \& Marttinen, 2017). Prior research in this area centered on propensity score methods (Bogard, 2013) and sometimes concentrated on theoretical models such as Soria, et al.'s (2017) application of Astin's (1997) theoretical model.

\section{Predictive Models}

Unlike normative models, predictive models may use student background data solely or in combination with self-report data. Researchers frequently use self-reported socio/psychological factors combined with background information, such as financial needs and support, high school academic performance, and student characteristics including demographics, media type usage, and geographic location (Wetzel, O’Toole, \& Peterson, 1999). Another common approach uses only background student data to predict student attrition (e.g., Bautista, 2016; Bogard, Helbig, Huff, \& James, 2011; Delen, 2011; Hanover, 2011; Raju \& Schumacker, 2015). While descriptive and normative models provide valuable insights regarding student attrition, researchers often choose predictor variables based on availability (Hausmann, et al., 2007). Caison (2007) found that student 
academic and background-based models outperformed a survey based self-report model in predicting attrition. In addition, identifying additional predicting variables from background and academic data may lead to further investigation of theoretical reasons for attrition (Delen, 2011) in descriptive and normative models.

Model prediction accuracy depends on the availability of student academic and background data. Based on student retention research using data collected from over 75,000 freshman constituting a response rate of 79.4\%, Astin (1997) concluded that the bulk of variance in retention could be explained by four student-based variables: high school GPA; admission test (SAT, ACT) scores; sex; and, race. He acknowledged additional significant predictors but emphasized that these four explained the largest part of the variance in retention. Powell (2003) supports Astin's findings, past and current academic performance (e.g., G.P.A.) best predicts student retention.

Descriptive and normative models offer rich models that provide underlying reasons for student attrition that enables effective intervention to decrease attrition rates. However, research in these theoretical areas lacks discussion of the resources to acquire the student self-response information. In addition, acquiring this information at freshmen orientation conflicts with the Target College's streamlined freshman orientation approach. While researchers use comprehensive predictive models (Wetzel, et al., 1999) or parsimonious predictive models (Bautista, 2016, and others) for their analysis. The literature is lacking in investigating a school that used a comprehensive predictive model and then downsized to a parsimonious predictive model. A comprehensive model provides a higher goodness of fit, or prediction accuracy, while a parsimonious model offers simplicity that makes the model easier to understand (Vandekerckhove, Matzke \& Wagenmakers, 2015). Focusing our study on a school that downsized from a comprehensive predictive model to parsimonious model provides an in-depth analysis of the tradeoff between simplicity and higher attrition prediction accuracy. 


\section{Statistical Modeling Options}

As the research on student attrition progresses, the variety of comparisons increases in researchers' attempts to improve predictability. These comparisons include underlying theories (Cabrera, et al., 1993; Kerby, 2015; Milem \& Berger, 1997), predictor variables (Herzog, 2005), and analytical methods (Bautista, 2016; Lin et al., 2009; Stage, 1988). Statistical modeling techniques tend to vary for predictive and descriptive models.

As the most popular modeling approach for predicting the probability of a certain behavior, logistic regression is favored for predicting the probability of each student dropping out (Bogard, 2011). From a predictive perspective, the logistic regression approach employs use of socio/ psychological factors combined with single-item directly measurable academic and background variables (Wetzel, et al., 1999). Dey and Astin (1993) and Lin, Imbrie, and Reid (2009) compare various attrition predictive models.

Researchers most frequently employ structural equation modeling (SEM) software when testing descriptive models (Stage, 1989). Researchers also use SEM to test normative models (Roland et al., 2018). SEM frequently uses multi-item measures of constructs while predictive forecast models such as logistic regression may use single-item or multi-item measures directly (Stage, 1988). For example, Stage (1988) specifies multi-item measures of constructs in a SEM to estimate parameters of the causal model using LISREL. He then adds the weightings from the measurement model to form single item measures (like factor scores) of social and academic integration for use in the logistic regression model to predict attrition (Stage, 1988).

Another modeling option involves types of model variables. Theory underlying retention models and the intended use of analysis results drives the choice of model variables. The early efforts to identify causes of attrition eventually led to structural models of related constructs. Building on theoretical models such as Tinto (1975), Bean (1980), and Dewberry and Jackson (2018), researchers establish validated scales 


\section{The BRC Academy Journal of Education Vol. 8, No. 1}

(e.g., IIS Scale of Pascarella \& Terenzini, 1983) to integrate models (e.g., Cabrera, et al., 1993; Milem \& Berger, 1997) using structural equation modeling (SEM) techniques.

In addition to SEM, researchers used various statistical methods to predict attrition. A sampling of statistical prediction methods include, but is not limited to; statistical learning, decision trees, random forest, boosting, generalized boosted models (GBM) (Bautista, 2016), neural networks, decision trees and ensemble models (Bogard, 2011; Delen, 2011; Dreiseitl \& Ohno-Machado, 2002). Prediction methods also include discriminant analysis, structural equation modeling (Lin et al, 2009), artificial neural networks, support vector machines, hierarchical Bayes (Stevens \& Taylor, 2009) and propensity scores (Bogard, 2013). The thirdparty firm, hired by Target College in 2016, used a logistic regression model supplemented by a tree-structure model similar to CHAID model (Kass, 1980).

At Target College scarce data and a limited number, and complexity, of variables led to simpler models with fewer variables. Simpler models, such as the one used in this study, use classification data such as achievement scores, demographic, academic, social and financial predictors (Astin, 1997; Hanover, 2011). Based on Target College's single item variables, we chose logistic regression for our attrition prediction model.

\section{MeTHODOLOGY}

Prior to applying logistic regression to Target College data, we needed to address the issue of an unavailable dependent variable. The data lacked the actual designation of each student as a freshman dropout or continuing student. In the rich data years, the data included a binary dropout designation variable for each student. In the absence of the dropout designation variable, the research study used the following binary dropout variable. Dropout was set to "persist" when the student both completed the previous semester and enrolled in the current semester, 
and set to "dropout" when the student completed the previous semester but did not enroll in the current semester. (cf., Stage 1988, p. 347). This served as the dependent variable, labeled dropout, in our logistic regression model.

\section{Limited Preview Complete}

This concludes the limited preview of this paper. Please purchase full access.

\section{Citation Information}

O’Donnell, Joseph B., and Paul L. Sauer. "When Big Data Becomes Little Data: Predicting Freshmen Attribution.” The BRC Academy Journal of Education 8, no. 1 (2020): 1-27. https://dx.doi.org/10.15239/ j.brcacadje.2020.08.01.ja01

\section{Web Appendix}

A web appendix for this paper is available at: https://dx.doi.org/10.15239/ j.brcacadje.2020.08.01.wa01 